\title{
Research Progress of Ultrasound Diagnosis in Early Breast Cancer
}

\author{
Yan Liu, Xinying Li, Ruizhi Hou \\ China-Japan Union Hospital of Jilin University, Changchun, Jilin Province, 130021
}

Keywords: Research Progress, Ultrasound Diagnosis, Early Breast Cancer

\begin{abstract}
Breast cancer is the most common malignancy in humans. Most patients have had regional lymph node metastasis when they are found, with a mortality rate of up to $50 \%$, whereas long-term cure rates for early-stage breast cancer can reach more than $90 \%$. Therefore, the early detection of breast cancer, early diagnosis and early treatment is the key to improving the cure rate and reduce mortality. This study retrospectively analyzed the ultrasonographic and spectral Doppler characteristics of early breast cancer, non-early breast cancer and benign breast tumor with diameters of less than or equal to $2.0 \mathrm{~cm}$, which were diagnosed by operation and pathology. Combined with the clinical mammography examination, X-ray examination explores the value of ultrasonic diagnosis in the diagnosis of early breast cancer.
\end{abstract}

\section{Introduction}

With the continuous improvement of people's living standards, breast cancer has become the highest incidence of malignant tumors in women, if the treatment is not timely and it is very easy to cause the patient to die. However, the long-term treatment of early breast cancer will greatly enhance the treatment rate. With the continuous development of medical treatment, the application of ultrasonic diagnosis technology in disease diagnosis is also more extensive, which can effectively diagnose the benign and malignant lesions. Therefore, in the treatment of breast cancer, ultrasound diagnostic techniques have been greatly applied to effectively diagnose early breast cancer and improve its cure rate. The following is the ultrasound examination of patients with breast lumps was analyzed.

\section{Materials and Methods}

A total of 108 patients with small breast mass received from January 2016 to January 2017 in our hospital were randomly selected as research materials. The tumor mass was less than $2 \mathrm{~cm}$ in diameter. According to the operation and pathology, 58 cases of benign lesions were included, ranging in age from 22 to 58 years (mean, $36.93 \pm 4.27$ years); early breast cancer group (50 cases, aged 22 to 60 years), The average age was $(36.25 \pm 4.18)$ years old. The comparative analysis of the basic data of the two groups showed no significant difference $(\mathrm{P}>0.05) .58$ cases of benign lesions and 50 cases of early breast cancer were examined by ultrasonic diagnostic techniques. PHLIPS-IU22, GE-E9, Mindray DC-8PRO, color Doppler ultrasound scanner, To $12.5 \mathrm{MHz}$. The probe placed above the breast, moderate pressure, with nipple as the center for longitudinal, transverse, oblique and other continuous scanning, the nipple as the center marker mass location, and to assess the distance between the mass and the nipple, the type of breast, Level, mass location, size, envelope, boundary, aspect ratio, internal echo, echo and so on [1].

Two-dimensional ultrasound imaging features and characteristics of ultrasound flow (blood flow classification, resistance index $>0.7$ ) were compared between the two groups. Imaging features include unclear boundary, crab legs syndrome, irregular shape, calcification of calculi, and weakening of posterior echo. Ultrasonic blood flow classification: no blood flow signal appears at grade 0; blood flow is reduced at grade I, only one or two punctate vessels or thin rodlike vessels can be glimpsed; grade II is more blood flow, and punctate vessels are easily observed; grade III is rich in blood flow and many important blood vessels or punctate spots can be observed Blood vessels, at least more than 5 . The data of this study were input into SPSS20.0 software, and normal 
measurement data were expressed as mean \pm standard deviation ( $\mathrm{x}- \pm \mathrm{s}$ ); normal count data was expressed as (\%); normal measurement data were $\mathrm{t}$ test; normal count data by chi-square test. $\mathrm{P}$ $<0.05$ statistically is significant difference.

\section{Results}

In group A, 327 cases of breast cancer were detected by ultrasound and 96 cases were missed. The diagnostic coincidence rate was $77.30 \%$ and the rate of misdiagnosis was $22.70 \%$. In group B, 139 cases were diagnosed as breast cancer by ultrasound and 6 cases were missed. The diagnostic rate was $95.86 \%$ \%, Misdiagnosis rate of 4.14\%. 33\%. Of the 1268 normal masses in group C, 93 cases were misdiagnosed as breast cancer by ultrasonography. The misdiagnosis rate was $7.33 \%$. Ultrasound sonography has seven major features, ultrasound characteristics of A, B group was no significant difference $(\mathrm{P}>0.05)$; $\mathrm{A}, \mathrm{C}$ group, the difference was statistically significant $(\mathrm{P}<0.05)$.

Of the 1836 breast lumps with diameter $\leq 2.0 \mathrm{~cm}$ confirmed by surgery and pathology, 568 were breast cancer and 1,268 were benign lumps. The former included 423 cases of early stage breast cancer (group A), including 186 cases of ductal carcinoma in situ, 139 cases of lobular carcinoma in situ and 98 cases of early invasive carcinoma. 145 cases of non-early stage breast cancer (group B), of which invasive ductal carcinoma 39 For example, medullary carcinoma in 20 cases, invasive lobular carcinoma in 46 cases, mucinous carcinoma in 25 cases, inflammatory reaction in 15 cases. A group of 57 cases of clinical surgical detection, missed diagnosis of 366 cases, the diagnostic rate of $13.48 \%$, the missed diagnosis rate of $86.52 \%$; B group of surgical patients was detected in 65 cases, 80 cases of missed diagnosis, the diagnosis rate was $44.83 \% 55.17 \%$. A total of 1268 benign breast masses (C group) included benign lesions including 743 cases of breast fibroadenoma, 442 cases of tumor-like hyperplasia, 59 cases of breast lymph nodes and 24 cases of breast tumor. The group of clinical surgical misdiagnosed as breast cancer may be 47 cases, the misdiagnosis rate was $3.70 \%$ [2]. Breast cancer patients (including early breast cancer group and non-early breast cancer group) and benign mass patients in the age $>40$ years of age, postmenopausal, family history 3 aspects, the difference was statistically significant $(\mathrm{P}<0.05)$ Liquid, nipple retraction, skin orange peel-like changes, $B$ group and $C$ group, the difference was statistically significant $(\mathrm{P}<0.05)$; breast thickening, breast lumps in both groups were compared, the difference was not statistically significant Significance ( $\mathrm{P}>0.05$ ); axillary lymph nodes large or fusion, $\mathrm{B}$ group and $\mathrm{C}$ group, the difference was statistically significant $(\mathrm{P}<0.05)$.

A group of 327 cases of X-ray molybdenum target examination, detected 236 cases of breast cancer, missed 91 cases, the detection rate was $72.17 \%$. B group of 132 cases of X-ray molybdenum target examination, detected 108 cases of breast cancer, missed diagnosis in 24 cases, the detection rate was $81.82 \% .692$ patients in group C underwent 682 X-ray mammography examinations, 32 cases were misdiagnosed as breast cancer. There was no significant difference between group $\mathrm{A}$ and group $B(P>0.05)$, but there was significant difference between group $A$ and $C(P<0.05)$, as shown in Table 3.2. 4 Ultrasonography, X-ray molybdenum Target examination, clinical surgical examination and the combination of the three evaluation of ultrasound examination, mammography $\mathrm{X}$-ray examination and clinical surgical joint examination for the diagnosis of early breast cancer was $96.93 \%$ (410/423 cases), with the individual examination The difference was statistically significant $(\mathrm{P}<0.05)$. Ultrasound examination, $\mathrm{X}$-ray molybdenum target examination and clinical surgical examination compared to the diagnostic accuracy was significantly different $(\mathrm{P}<0.05)$.

\section{Discussions}

This study a group of 423 cases of early breast cancer palpable palpable mass in 57 cases, the clinical surgical examination diagnostic coincidence rate was only $13.48 \%$, missed diagnosis rate of $86.52 \%$, but not early breast cancer group (B Group) also had a diagnosis rate of $44.33 \%$ and a missed diagnosis rate of $55.17 \%$. Other literatures also pointed out that simple surgical examination cannot improve the detection rate of breast cancer [3]. The reason, the more obese patients, the larger the breast, the more dense the texture, the smaller the breast mass, the distance from the skin, 
the lower the detection rate of clinical surgery. Breast mammography X-ray examination can identify a variety of soft tissue within the breast and abnormal density changes. Typical X-ray findings of breast cancer: irregular mass, blurred edges or burrs, high density, fine needle-like or rod-like calcification. In the United States and many western countries routinely used for screening for breast cancer over the age of 50 . The domestic literature also reported its diagnosis of breast cancer in $67 \%$ to $80 \%$. In this study, mammography diagnostic accuracy of $72.17 \%$, it is also commonly used in clinical practice. However, most Oriental women are dense glands and the breasts are flat, so the development is poor. The posterior wall of the masses and the early stage breast cancer without obvious calcification may be missed [4] and have some radiation and are troublesome to operate, so not easy to frequent examination, so should be cautious in clinical use. Ultrasound diagnosis is a combination of high-frequency probe and color Doppler flow imaging. Rely on its high resolution to detect due to breast tissue due to adhesions, pull the lesions caused by irregular shape, edge irregularities in the measurement of the aspect ratio and found that the tip-like calcification points were single or cluster; and more Pulmonary blood flow imaging showed the lesion perfusion and blood flow characteristics. In this study, we can see the abnormal sonogram in 1836 breast masses with diameter less than $2.0 \mathrm{~cm}$, which can be seen and removed in the pathological examination. It is of far-reaching significance to understand and explain the disease, to be free from the above-mentioned burden, save a large amount of medical resources, and even improve the spiritual quality of the next generation. In short, recurrent spontaneous abortion is the result of the interaction between various factors. According to the analysis of the results of this study, multiple psychological factors such as spontaneous abortion, advanced age and gestational mental stimulation have the highest risk factors in recurrent spontaneous abortion. Therefore, paying attention to the influence of social psychological factors and adopting positive psychological interventions are of great significance for the effective prevention of recurrent spontaneous abortion. Prenatal anxiety has a significant impact on pregnancy, childbirth and the growth and development of offspring, which may lead to maternal and child adverse outcomes, and less attention is paid to the neurological behavior of the newborn in China. The purpose of this study was to investigate the specific risk factors of prenatal anxiety in recurrent spontaneous abortion and normal pregnant women, the effect of prenatal anxiety on delivery outcomes, and the relationship between prenatal anxiety and neonatal weight and neonatal behavioral neurology.

Modern medical, mammography can detect and identify timely breast a variety of soft tissue abnormalities and density changes. Breast cancer, the use of breast molybdenum target X-ray lesion detected more typical is the appearance of irregular mass shadow, the edge of the tumor appears fuzzy or burr phenomenon, with a high density, accompanied by fine needle Like or stick-like calcification phenomenon. In western countries, breast mammography is often used to screen breast cancer patients over the age of 50. In our country's relevant literature, it has also been reported for the diagnosis of breast cancer rate of $67 \%$ to $80 \%$. In this paper, the study of mammography X-ray diagnosis coincidence rate is about $72 \%$. Therefore, the mammography can be used in mammography in clinical examination. However, the majority of women in the Oriental countries showed dense breasts with flattened glands and relatively poor imaging. The tumors also showed some misdiagnosis in the early stage of breast cancer with no obvious calcification. At the same time as the breast mammography X-ray there will be a certain degree of radiation in the actual operation is relatively cumbersome, in practice, it is not easy to carry out frequent checks, it is necessary to be careful mammography [5]. Clinically, ultrasound is a composite technique that uses a higher frequency probe combined with color Doppler flow phenomena. Mainly rely on the high resolution of ultrasound diagnostic techniques to detect the edge of the lesion in the breast due to tissue adhesion or traction. By measuring its aspect ratio, single or clustered appearances of the tip-like calcifications can be detected and Doppler flow imaging can show perfusion and characteristics of the lesion.

\section{Conclusions}

Breast cancer is a very serious disease for women, which poses a great threat to the life safety of 
women. The treatment of breast cancer should be checked and treated promptly in time to improve the cure rate. With the continuous development of medical treatment, the continuous improvement of ultrasonic diagnostic technology, the use of ultrasonic diagnostic technology in the detection of early breast cancer is a very effective way, in the diagnosis of early breast cancer has a great advantage, with a high degree of precision, timely detection of where the illness, the diagnosis, so that patients with timely treatment.

\section{References}

[1] Kramer MS, Lydon J, Séguin L, et al. Stress pathways to spontaneous preterm birth: the role of stressors, psychological distress, and stresshormones [J]. Am J Epidemiol, 2009, 169 (11): 1319-1326.

[2] Hilmert CJ, Schetter CD, Dominguez TP, et al. Stress and blood pressure during pregnancy: racial differences and associations with birth weight [J]. Psychosomatic Medicine, 2008, 70 (1): 57-64.

[3] Beddoe AE, Lee KA. Mind-Body Interventions during pregnancy[J]. JOGNN, 2008, 37 (2): 165-175.

[4] Austin MP, Hadzi-Pavlovic D, Leader L, et al. Maternal trait anxiety, depression and life event stress in pregnancy: relationships with infant temperament [J]. Early Hum Dev, 2005, 81 (2): 183-190.

[5] Diego MA, Jones NA, Field T, et al. Maternal psychological distress, prenatal cortisol and fetal weight [J]. Psychosom Med, 2006, 68 (5): 747-753.

[6] Van der wal MF, van Eijsden M, Bonsel GJ. Stress and emotional problems during pregnancy and excessive infant crying [J]. Dev Behav Pediatr, 2007, 28 (6): 431-437. 